\title{
Critical pitch angle for electron acceleration in a collisionless shock layer
}

\author{
Y. Narita $^{1}$, H. Comişel ${ }^{2,3}$, and U. Motschmann ${ }^{2,4}$ \\ ${ }^{1}$ Space Research Institute, Austrian Academy of Sciences, Schmiedlstr. 6, 8042 Graz, Austria \\ ${ }^{2}$ Institut für Theoretische Physik, Technische Universität Braunschweig, Mendelssohnstr. 3, 38106 Braunschweig, Germany \\ ${ }^{3}$ Institute for Space Sciences, Atomiştilor 409, P.O. Box MG-23, Bucharest-Măgurele 077125, Romania \\ ${ }^{4}$ Deutsches Zentrum für Luft- und Raumfahrt, Institut für Planetenforschung, Rutherfordstr. 2, 12489 Berlin, Germany
}

Correspondence to: Y. Narita (yasuhito.narita@oeaw.ac.at)

Received: 1 May 2016 - Revised: 14 June 2016 - Accepted: 29 June 2016 - Published: 12 July 2016

\begin{abstract}
Collisionless shock waves in space and astrophysical plasmas can accelerate electrons along the shock layer by an electrostatic potential, and scatter or reflect electrons back to the upstream region by the amplified magnetic field or turbulent fluctuations. The notion of the critical pitch angle is introduced for non-adiabatic electron acceleration by balancing the two timescales under a quasi-perpendicular shock wave geometry in which the upstream magnetic field is nearly perpendicular to the shock layer normal direction. An analytic expression of the critical pitch angle is obtained as a function of the electron velocity parallel to the magnetic field, the ratio of the electron gyro- to plasma frequency, the cross-shock potential, the width of the shock transition layer, and the shock angle (which is the angle between the upstream magnetic field and the shock normal direction). For typical non-relativistic solar system applications, the critical pitch angle is predicted to be about $10^{\circ}$. An efficient acceleration is expected below the critical pitch angle.
\end{abstract}

Keywords. Space plasma physics (charged particle motion and acceleration; shock waves)

\section{Introduction}

Collisionless shocks in space and astrophysical plasmas are unique in that electrons are efficiently accelerated there. Different types are possible for the acceleration mechanisms. For a quasi-parallel shock at which the upstream magnetic field is nearly aligned with the normal direction of the shock layer, the electrons can be efficiently trapped by turbulent fluctuations on the both sides of the shock. Since the incom- ing flow speed on the upstream side is higher than that on the downstream side, the electron trapping leads to a diffusive shock acceleration (Drury, 1983). For a quasi-perpendicular shock, the electrons are accelerated within the shock transition layer by the electrostatic, cross-shock potential which is sustained by different bulk motions of the ions to the electrons (Goodrich and Scudder, 1984). On the other hand, the electrons can be scattered or reflected away from the shock layer by a sudden increase of the magnetic field or turbulent fluctuations at the shock ramp. One may thus formulate that the electrons at the quasi-perpendicular shock undergo two competing effects: acceleration or scattering. The purpose of this article is to estimate the critical pitch angle for the electron acceleration at the quasi-perpendicular shock.

\section{Critical pitch angle}

Naively speaking, for a smaller pitch angle, the electrons are nearly field-aligned (with respect to the magnetic field) and should have sufficient time to stay in the shock transition layer for an efficient acceleration by the electrostatic potential. For a larger pitch angle, the electrons are scattered and eventually kicked away from the shock transition layer, either in the upstream and downstream directions. We define the critical pitch angle through the balance between two timescales, the electron acceleration time $\tau_{\text {acc }}$ and the electron scattering time $\tau_{\mathrm{sc}}$, into a formula as $\tau_{\mathrm{acc}}=\tau_{\mathrm{sc}}$.

One may roughly estimate the acceleration timescale as $\tau_{\text {acc }}=\frac{\ell}{v_{\|}}$, where $\ell$ stands for the field-aligned length and $v_{\|}$the electron velocity parallel to the magnetic field, respectively. Likewise, the scattering timescale is estimated as 
$\tau_{\mathrm{sc}}=\frac{d}{v_{\perp}}$ using $d$, which is the width of the shock transition layer and $v_{\perp}$ the perpendicular velocity. The two length scales, $\ell$ and $d$ are related to each other by an angle $\theta_{\mathrm{nB}}$ between the upstream magnetic field and the shock normal direction as $\ell \cos \theta_{\mathrm{nB}}=d$.

We use the equation of motion (in a non-relativistic sense) for the electron in the parallel or field-aligned component:

$m \dot{v}_{\|}=e E_{\|}$,

from which the parallel velocity for a timescale of $\tau_{\text {acc }}$ is obtained as

$v_{\|}=\frac{e}{m} E_{\|} \tau_{\mathrm{acc}}+v_{\| 0}$

and the length scale parallel to the field as

$\ell=\frac{e}{m} E_{\|} \tau_{\mathrm{acc}}^{2}+v_{\| 0} \tau_{\mathrm{acc}}$.

Here $m$ denotes the electron mass, $e$ the electric charge of the electron, $E_{\|}$the field-aligned component of the electric field, and $v_{\| 0}$ the initial parallel velocity of the electron. For the perpendicular component, we have

$d=v_{\perp} \tau_{\mathrm{sc}}$

That is, the scattering timescale is of the order of the electron gyro-period. The pitch angle is defined as $\tan \alpha=v_{\perp} / v_{\|}$. The electrons are treated as non-adiabatic particles in this theoretical frame by neglecting the mirror force in Eq. (1) and the electron drift in Eq. (4). See Eqs. (3.92) and (3.87) in Bellan (2006) for the parallel component of equation of motion and the perpendicular component of electron drift, respectively. The question of whether the electrons are adiabatic or not at the shock transition needs to be evaluated additionally when testing the notion of the critical pitch angle against observational or simulation data.

The acceleration timescale can explicitly be obtained by regarding Eq. (3) as a quadratic equation of $\tau_{\text {acc }}$. The solution for $\tau_{\text {acc }}>0$ is

$$
\tau_{\mathrm{acc}}=\frac{m}{2 e E_{\|}}\left(-v_{\| 0}+\sqrt{v_{\| 0}^{2}+4 \frac{e}{m} E_{\|}} \ell\right) .
$$

The condition for the critical pitch angle is $\tau_{\mathrm{acc}}=\tau_{\mathrm{sc}}$, from which we obtain an estimate for the critical pitch angle $\alpha_{\mathrm{cr}}$ (after some algebra) as

$$
\begin{aligned}
\tan \alpha_{\mathrm{cr}} & =\frac{2 e E_{\|} d}{m v_{\| 0}^{2}}\left[-1+\sqrt{1+\frac{4 e E_{\|} d}{m v_{\| 0}^{2} \cos \theta_{\mathrm{nB}}}}\right]^{-1} \\
& =\frac{2 x}{-1+\sqrt{1+\frac{4 x}{\cos \theta_{\mathrm{nB}}}}},
\end{aligned}
$$

where the dimensionless quantity $x$ is introduced as

$x=\frac{e E_{\|} d}{m v_{\| 0}^{2}}=\frac{c^{2}}{v_{\| 0}^{2}} \frac{\Omega_{\mathrm{e}}}{\omega_{\mathrm{pe}}} \frac{E_{\|}}{B} \frac{d}{\lambda_{\mathrm{e}}}=\frac{c^{2}}{v_{\| 0}^{2}} \frac{\Omega_{\mathrm{e}}}{\omega_{\mathrm{pe}}} \frac{\Phi}{B \lambda_{\mathrm{e}}} \frac{d}{\lambda_{\mathrm{e}}}$.

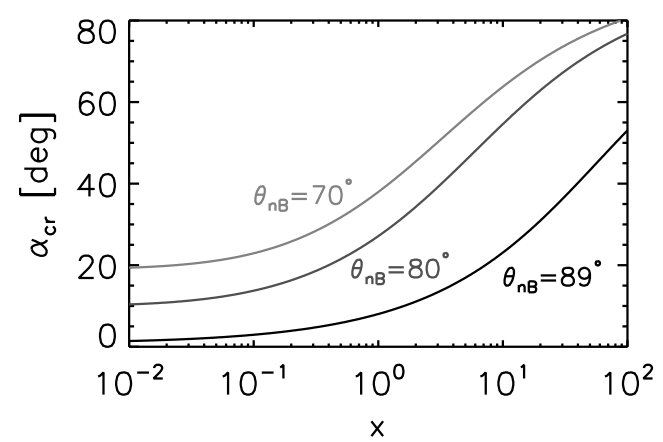

Figure 1. Critical pitch angle as a function of the dimensionless quantity $x$ for different values of the shock angle between the upstream magnetic field and the shock normal direction $\theta_{\mathrm{nB}}$.

For the particle-in-cell simulation by Comişel et al. (2011, 2015), $\frac{v_{\| 0}}{c}=0.3, \frac{\Omega_{\mathrm{e}}}{\omega_{\mathrm{pe}}}=\frac{1}{8}, \frac{\Phi}{B \lambda_{\mathrm{e}}}=0.1, \frac{d}{\lambda_{e}}=1, \theta_{\mathrm{nB}}=81^{\circ}$, the dimensionless quantity is $x=0.14$, and the critical angle is $\alpha_{\mathrm{cr}}=14^{\circ}$.

Figure 1 shows the profile of the critical pitch angle $\alpha_{\mathrm{cr}}$ as a function of the dimensionless quantity $x$ (Eq. 7). Smaller values of $x$ indicate high-energy, small-pitch-angle electrons in a thin shock layer. The critical pitch angle shows an asymptotic behavior at smaller values of $x(x<0.1)$, and becomes smaller from about 20 to $10^{\circ}$ and further to $1^{\circ}$ as the magnetic field angle from the shock normal changes from 70,80 , and $89^{\circ}$. It is interesting to note that $x$ increases at a larger electrostatic potential and a weaker magnetic field. Therefore, a stronger electrostatic potential leads to a larger critical pitch angle and thus enables more particles to be accelerated.

\section{Perspective}

The critical shock angle is formulated as a function of the electron velocity parallel to the magnetic field, the ratio of the electron gyro- to plasma frequencies, the electrostatic potential, the width of the shock transition layer, and the shock angle (which is the angle between the upstream magnetic field and the shock normal direction). From the above simple (and algebraic) estimate, particularly in Eq. (8), one draws a lesson that the electron acceleration is efficient in an increasing sense of the critical pitch angle (or equivalently, an increasing sense of the dimensionless quantity $x$ ) under the conditions of (1) lower parallel velocities of the incoming particles, (2) a stronger magnetic field or a lower electron density, (3) a higher electrostatic potential at the shock transition, or (4) a thicker transition layer. For typical non-relativistic solar system applications, the critical pitch angle is predicted to be about $10^{\circ}$ or even less. Our treatment does not include the effect of turbulent fluctuations explicitly. Thus it would be interesting to study in detail whether or how adiabatic electron 
acceleration and heating are affected by the shape or time evolution of the shock potential.

A caveat needs to be addressed here. As we have assumed non-adiabatic motion of electrons, the spatial gradient of the magnetic or electric field must be smaller than the electron gyro-radius such that the magnetic moments of electrons are no longer constant. The condition of non-adiabatic motion for the electric field is (Gedalin et al., 1995)

$\frac{e\left|\nabla E_{\perp}\right|}{m_{\mathrm{e}} \Omega_{\mathrm{e}}^{2}}>1$.

Here $\Omega_{\mathrm{e}}=e B / m_{\mathrm{e}}$ is the electron cyclotron frequency. Using the Gauss law on the electric field,

$\nabla \cdot \boldsymbol{E}=\frac{\rho_{\mathrm{e}}}{\epsilon_{0}}$

where $\rho_{\mathrm{e}}$ and $\epsilon_{0}$ denote the charge density and the permittivity of free space, respectively. One may re-write the nonadiabatic condition (Eq. 9) into a simpler form (using the relation to the permeability of free space $\mu_{0}$ and the speed of light $c$ as $\epsilon_{0} \mu_{0}=c^{-2}$ ) as

$$
\frac{\mathcal{E}_{\text {rest }}}{\mathcal{E}_{\text {mag }}}=\frac{\frac{1}{2} m_{\mathrm{e}} \delta n_{\mathrm{e}} c^{2}}{\frac{1}{2 \mu_{0}} B^{2}}>1,
$$

where the fluctuating electron number density is estimated through the electric charge density as $\delta n_{\mathrm{e}}=\rho_{\mathrm{e}} / e$, neglecting the ion contribution. In essence, the rest energy of the perturbed electron fluid must exceed the energy density of the magnetic field.

Understanding the relationship between the cross-shock potential and the particle dynamics such as trapping, parallel acceleration, and perpendicular scattering with respect to the mean magnetic field has various applications to the collisionless shocks in the solar system and in astrophysical systems. The method of Liouville mapping provides hightime-resolution electron velocity distribution functions and is a useful tool to evaluate the cross-shock potential (Scudder et al., 1986; Scudder, 1995; Lefebvre et al., 2007). The dependence of the critical pitch angle on the shock geometry, the shock potential, and the electron parallel velocity can be tested not only numerically using direct numerical simulations such as particle-in-cell algorithms but also observationally using the novel MMS (Magnetospheric Multiscale) mission (Burch et al., 2016). It is also interesting to note that the idea of critical pitch angle works for any short-scale gradient with a parallel electric field such as a steepened electronscale whistler wave, and not exclusively at shock waves.

Acknowledgements. H. Comişel thanks Manfred Scholer and Octav Marghitu for stimulating and useful discussions which led to developing and formulating the notion of the critical pitch angle for this article. The work by H. Comişel and U. Motschmann in Braunschweig is supported by an extended program of Collaborative
Research Center 963, "Astrophysical Flow, Instabilities, and Turbulence" of the German Science Foundation. The work conducted by H. Comişel in Bucharest is supported by Romanian Ministry for Scientific Research and Innovation, CNCS - UEFISCDI, project number PN-II-RU-TE-2014-4-2420.

Edited by: C. Owen

Reviewed by: one anonymous referee

\section{References}

Bellan, P. M.: Fundamentals of Plasma Physics, Cambridge University Press, Cambridge, 2006.

Burch, J. L., Moore, T. E., Torbert, R. B., and Giles, B. L.: Magnetospheric multiscale overview and science objectives, Space Sci. Rev., 199, 5-21, doi:10.1007/s11214-015-0164-9, 2016.

Comişel, H., Scholer, M., Soucek, J., and Matsukiyo, S.: Nonstationarity of the quasi-perpendicular bow shock: comparison between Cluster observations and simulations, Ann. Geophys., 29, 263-274, doi:10.5194/angeo-29-263-2011, 2011.

Comişel, H., Narita, Y., and Motschmann, U.: Adaptation of the de Hoffmann-Teller frame for quasi-perpendicular collisionless shocks, Ann. Geophys., 33, 345-350, doi:10.5194/angeo-33345-2015, 2015.

Drury, L. O'C.: An introduction to the theory of diffusive shock acceleration of energetic particles in tenuous plasmas, Rep. Prog. Phys., 46, 973-1027, doi:10.1088/0034-4885/46/8/002, 1983.

Gedalin, M., Gedalin, K., Balikhin, M., Krasnosselskikh, V., and Woolliscroft, L. J. C.: Demagnetization of electrons in inhomogeneous $E \perp B$ : Implications for electron heating in shocks, J. Geophys. Res., 100, 19911-19918, doi:10.1029/95JA01399, 1995.

Goodrich, C. C. and Scudder, J. D.: The adiabatic energy change of plasma electrons and the frame dependence of the cross-shock potential at collisionless magnetosonic shock waves, J. Geophys. Res., 89, 6654-6662, doi:10.1029/JA089iA08p06654, 1984.

Lefebvre, B., Schwartz, S. J., Fazakerly, A. F., and Décréau, P.: Electron dynamics and cross-shock potential at the quasiperpendicular Earth's bow shock, J. Geophys. Res., 112, A09212, doi:10.1029/2007JA012277, 2007.

Scudder, J. D.: A review of the physics of electron heating at collisionless shocks, Adv. Space Res., 15, 181-223, doi:10.1016/0273-1177(94)00101-6, 1995.

Scudder, J. D., Aggson, T. L., Mangeney, A., Lacombe, C., and Harvey, C. C.: The resolved layer of a collisionless, high beta, super-critical, quasi-perpendicular shock wave: I. RankineHugoniot geometry, currents, and stationarity, J. Geophys. Res., 91, 11019-11052, doi:10.1029/JA091iA10p11019, 1986. 